
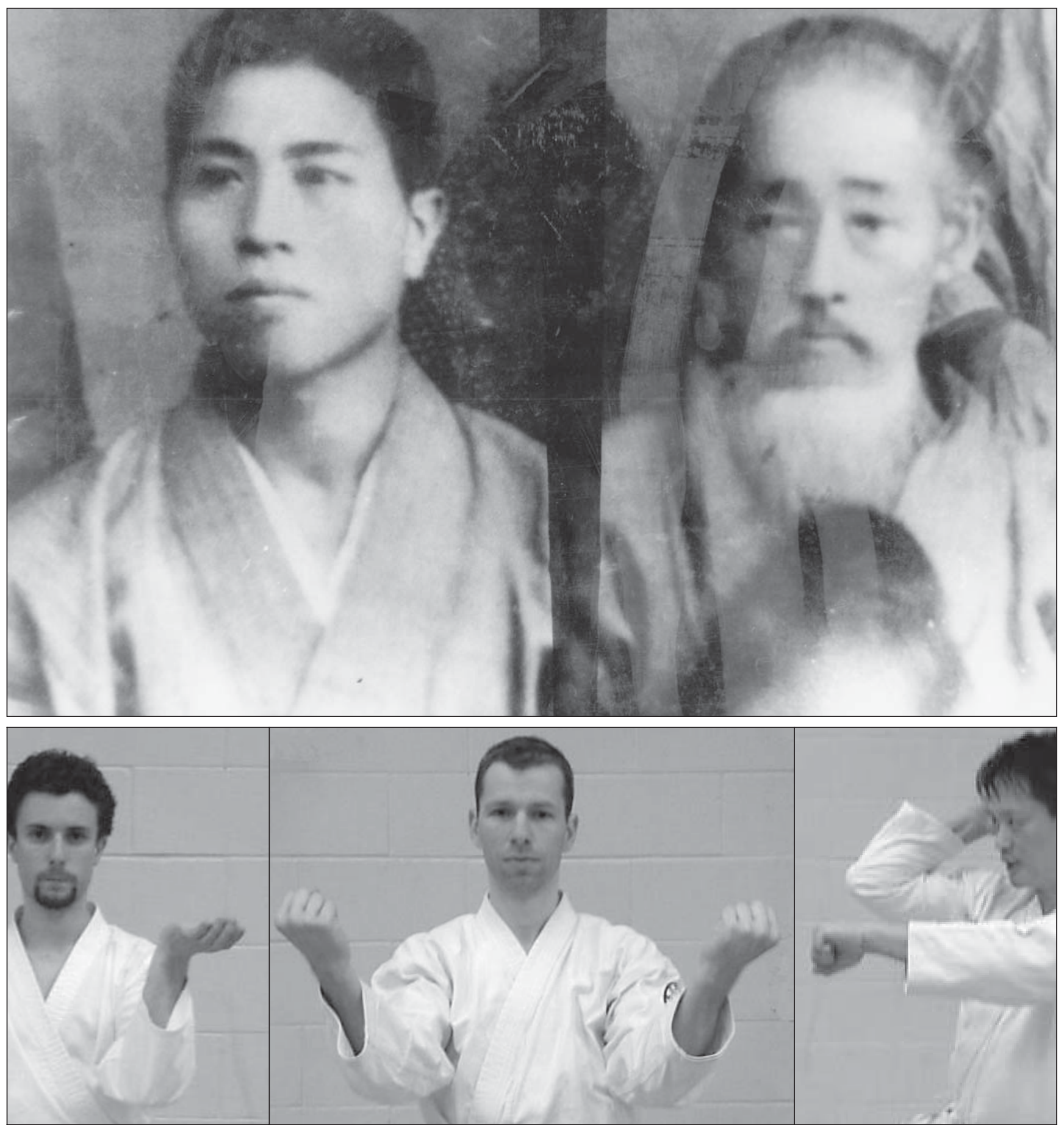

ARribA:

\title{
Resumen
}

HigaONnA KanRYO

Miyagi Chojun ( 1888 -1953) fue el fundador del Goju-ryu, un estilo de kárate-do que supuestamente Y SU ESTUDIANTE tenía lazos con una escuela de boxeo chino establecida en Okinawa en 1828. Miyagi se basó en las

KYODA JUHATSU enseñanzas de su primer instructor, Higaonna Kanryo (1850-1915). Hasta ahora, la transmisión del (IZQUIERDA)

AваJо: actual Goju-ryu en lo que se refiere a sus katas (formas) y estas fuentes históricas nunca ha sido establecida. Por ello, hemos utilizado una técnica estadística para analizar la relación entre las katas clásicas del Goju-ryu moderno. Se ha concluido que podría existir esta continuidad a través de todas las katas del Goju-ryu moderno si en verdad éstas vinieron de una fuente similar. Los resultados obte-

TRES POSTURAS CLÁSICAS nidos son esclarecedores y sugieren los posibles orígenes de las katas Goju-ryu. 


\section{UN ANÁLISIS PRELIMINAR DE LA ESTRUCTURA DE}

\section{LA Kata GOJU-RYU}

Fernando Portela Cámara \& Mario Mckenna

$\Delta \nabla \mathbf{\Delta}$

\section{Introducción}

Goju-ryu es el nombre de un estilo de karate-do que Miyagi Chojun estructuró en la década de 1930. Miyagi afirmaba que su sistema se originó a partir de una escuela de quanfa chino (gong-fu) establecida en la ciudad de Naha en 1828 (Miyagi, 1934) y reconoció a Higaonna Kanryo (1853-1917) como la fuente primaria del sistema. No obstante, cuando analizamos el Goju-ryu moderno podemos ver diversas influencias importantes en su desarrollo. Éstas incluyen a) el método de kárate moderno desarrollado por Itosu Anko (1830-1916), b) la práctica tradicional y autóctona denominada Mano de Okinawa ( $\mathrm{Ti}$ o $\mathrm{Te}$ ), y c) los estudios personales de Miyagi ${ }^{1}$. Desarrollaremos brevemente cada una de estas influencias más abajo.

Itosu enseñó sus primeras clases de kárate públicamente como parte del currículo de educación física de las escuelas elementales en 1901, y más tarde en la escuela intermedia (Iwai, 1992; Consejo de Educación de la Prefectura de Okinawa, 1994)2 ${ }^{2}$. El método de Itosu incluía una serie de puntos clave (Kinjo, 1999; Murakami, 1991):

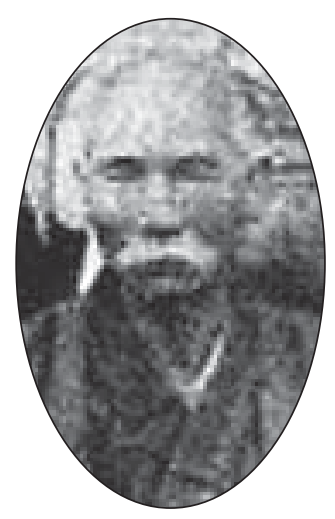

ITOSU ANKO

- LA PRINCIPAL PERSONA RESPONSABLE DE LA MODERNIZACIÓN DEL KÁRATE-DO

I) El desarrollo de un nuevo conjunto de katas introductorias llamadas Pinan,

2) La estandarización de las katas existentes,

3) El énfasis en la disciplina física y moral,

4) El cultivo de la ética, $y$

5) La des-enfatización de la pura técnica de combate (Kinjo, 1999; Murakami, 1991).

El método de Itosu fue seguido y mejorado por los sucesivos estudiantes y maestros como Funakoshi Gichin, Chibana Chosin, y Mabuni Kenwa, formándose los cimientos del kárate moderno de Okinawa y Japón.

La influencia del método de Itosu sobre Miyagi fue crucial para el futuro desarrollo del Goju-ryu. De acuerdo con Kinjo Hiroshi, el Goju-ryu es un estilo moderno en línea con el modelo de kárate de Itosu (Kinjo, 2007). Kinjo defiende que tanto Itosu como Higaonna estructuraron el kárate en 1905, y sin embargo sólo el modelo de Itosu fue adoptado por el Departamento de Educación de la Prefectura de Okinawa. Se rumoreó que esto fue debido al énfasis de Higaonna en el entrenamiento de la kata Sanchin, que era considerada inadecuada para el desarrollo de los adolescentes (Kinjo, 2007). Esto, junto con otro rumor existente sobre que Higaonna bebía excesivamente detuvo la introducción del kárate de Higaonna en el sistema escolar. Miyagi era un estudiante en la primera escuela intermedia de la prefectura durante la época en que se introdujo el modelo de kárate de Itosu, y habría aprendido kárate de Itosu y de su discípulo Chomo Hanashiro.

Todas las fotografías son cortesía de Mario McKenna. 


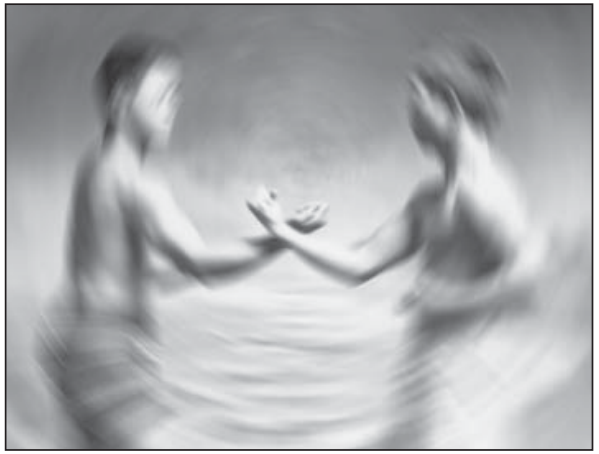

Un joven Miyagi Chojun

(IZQUIERDA) Y

KYODA JUHATSU

DEMOSTRANDO TÉCNICAS.

Ya adulto, Miyagi organizó el kárate que había aprendido de Higaonna y éste fue aceptado como parte de la escuela comercial de la prefectura de Okinawa. Este fue el kárate de Miyagi que más tarde llamaría Goju-ryu. Es interesante especular si una de las motivaciones de Miyagi para introducir el Goju-ryu en el sistema escolar fue el recuerdo de la amargura de su maestro y la temprana popularidad del kárate de Itosu.

En contraste con el karate-do, Ti es un termino genérico del periodo anterior a la Segunda Guerra Mundial relacionado con los ejercicios que no son kata basados en las tradiciones de combate sin armas practicadas en Okinawa (Hokama, 1999). No se refiere a un método, sistema o estilo de combate específico. La tradición oral de los maestros de kárate de Okinawa más ancianos se refiere a posiciones, posturas, técnicas, etc. con el término genérico Ti. Interesantemente, y de acuerdo con Morio Higaonna, Miyagi Chojun sólo se refería a lo que enseñaba como Ti (Higaonna, 1998).

Por último, existen varios, pero poco documentados, informes sobre la investigación de Miyagi Chojun de otros sistemas de lucha. Cuando se investiga la vida de Miyagi Chojun, observamos que empleó la mayor parte de su energía vital y la fortuna de su familia en estudiar las artes de combate. Durante sus estudios estuvo en contacto con tradiciones de combate tales como el Boxeo de la Grulla Blanca de Fujian (Baihe Quan), el Boxeo del Tigre (Hu Quan), el Boxeo del Puño del Monje (Lohan Quan), y posiblemente el Boxeo del Puño de los Cinco Ancestros (Wu Zu Quan) (Kinjo, 1999; Tokashiki, 1991). Esto suscita dudas en relación a la continuidad en las katas transmitidas por Miyagi Chojun.

\section{Catálogo de las katas Goju-ryu}

A finales de la década de 1930, el Goju-ryu constaba aparentemente de ocho katas: Seiunchiun, Saifa, Shisochin, Sanseru, Seipai, Seisan, Kururunfa, y Suparimpei / Pechurin; y dos ejercicios básicos que fueron adaptados en un formato de kata, Sanchin y Tensho. A principios de los años 40 se añadieron dos katas básicas introductorias: Gekisai I y II.

Kanzaki Shigekazu (nacido en 1928) y Murakami Katsumi (nacido en 1927) fueron discípulos de Kyoda Juhatsu (1887-1968), un estudiante durante largo tiempo de Higaonna y del padre de Miyagi. Ambos afirman que Higaonna Kanryo sólo enseñó las katas Sanchin, Seisan, Sanseru, y Pechurin. Analizando estas cuatro formas se revela que éstas tienen un patrón asimétrico. Esto es, las patadas se realizan predominantemente con la pierna derecha y las técnicas del tren superior se realizan frecuentemente con una sola parte del cuerpo.

Las katas Seiunchin, Saifa, Shisochin, Seipai, y Kururunfa son destacables en comparación con las cuatro katas mencionadas anteriormente, ya que son formas simétricas. Las patadas y las técnicas se realizan frecuentemente con ambos lados del cuerpo. Seiunchin, Seipai, y Kururunfa son mencionadas a finales de los años 30 en los escritos de Mabuni Kenwa (1889-1951), un maestro de kárate y amigo de Miyagi. Los primeros estudiantes de Miyagi también mencionan Seiunchin como una de las katas más antiguas que enseñaba. En contraste, Shisochin y Saifa no son mencionadas en ninguna lista de kárate publicada en las décadas de los años 20 y 30 . Curiosamente, parece que Miyagi fue la única persona que transmitió estas cinco katas.

\section{Fuentes alternativas}

Parece existir una falta de documentación escrita sobre el origen y transmisión de las katas Goju-ryu. Además, los testimonios de los antiguos maestros están llenos de contradicciones e inconsistencias. Tomando esto en consideración, los orígenes y el linaje de las katas Goju-ryu permanecen poco claros. 
De acuerdo con el ya citado historiador de artes marciales Hokama Tetsuhiro (Hokama, 1999), hay seis maneras de analizar las katas de kárate-do de Okinawa:

I) Métodos de desplazarse, utilización de las manos, y la utilización de la técnica, e.g. Sanchin, Shisochin;

2) Nombres de los fundadores o creadores de una tradición especial, e.g. Kusanku, Wansu, etc.;

3) Nombres de áreas específicas o distritos en los que se practicaba la tradición, e.g. Shuri, Naha, Tomari;

4) Los principios religiosos o espirituales inherentes dentro de la tradición, e.g. Suparimpei, Seipai;

5) Los aspectos metafísicos o transcendentales de una tradición (catarsis / purificación / Zen), e.g. Sanchin, Tensho; y

6) Los movimientos implícitos de animales.

De estos seis métodos, el análisis de los métodos de desplazarse, la utilización de las manos, y la utilización de la técnica en las katas Goju-ryu puede proporcionar unos medios interesantes y útiles para investigar su origen y evolución. En este artículo se analizarán las estructuras de las ocho katas de Goju-ryu mediante la técnica estadística exploratoria del análisis de conglomerados, con el objeto de obtener una clasificación de estas katas y formular ideas sobre sus orígenes.

\section{Extracción de información}

La extracción de información puede ser definida como la ciencia de obtener información útil de grandes conjuntos de datos. En otras palabras, la extracción de información proporciona un medio práctico para la clasificación y distinción de grandes cantidades de datos. Podemos imaginar la extracción de información utilizando una analogía con la extracción de oro. Un "minero de datos" busca "oro" (conocimiento útil e información) al "golpear el mineral" (montañas de datos) utilizando diferentes tipos de herramientas (tales como una técnica estadística). Por tanto, la extracción de información es una técnica que nos ayuda a distinguir el oro del mineral.

Para que nosotros podamos "extraer los datos" contenidos en las katas Goju-ryu, hemos clasificado cada kata sobre la base de la repetición de patrones o a la temática encontrada en ellas. Las katas Goju-ryu muestran una repetición de patrones que puede ser utilizada con un inventario para su clasificación. Por ejemplo, se pueden observar las siguientes características en las katas originales de Higaonna (Sesan, Sanseru, y Pechurin/Suparimpei), como ya expusieran Kanzaki y Murakami:

1) Todas comienzan con tres pasos hacia delante desde el ejercicio de Sanchin,

2) Todas tienen un patrón de realización en cuatro direcciones (o cruz),

3) Dos de ellas (Seisan y Pechurin/Suparimpei) tienen tres pasos en la posición de Sanchin mientras se realizan bloqueos dobles con las manos abiertas (osae-uke y sukui-uke).

Además, estas katas contienen asimetrías:

1) Las patadas se realizan con la pierna derecha;

2) Los golpes se concluyen con el puño izquierdo.

En contraste, Saifa, Shisochin, Seipai, Kururunfa, y Seiunchin son katas simétricas. Shisochin, Saifa, y Seiunchin comienzan con tres pasos de avance mientras se repite la misma técnica, pero no siguen la postura o patrón Sanchin. La mayoría de las técnicas de estas cinco katas se realizan con ambos lados del cuerpo, ejecutándose de un modo simétrico. 


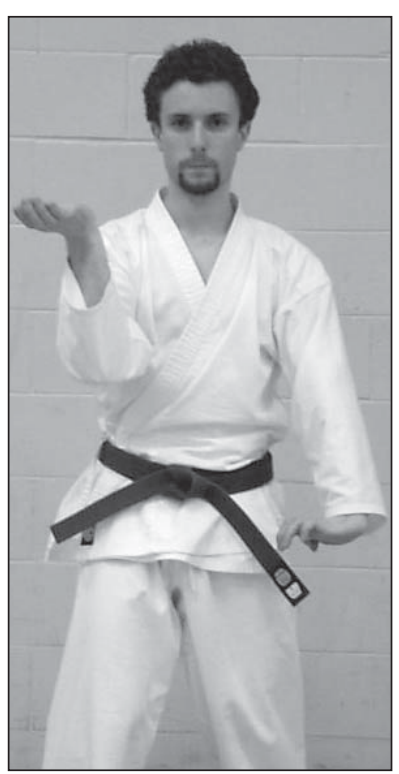

BLOQUEO EN FORMA DE CUCHARA (SUKUI UKE).
Basándose en estas observaciones, dos veteranos instructores de Goju-ryu identificaron diez temáticas entre las katas Goju-ryu. Luego cada kata fue valorada por los instructores por la presencia (1) o ausencia (0) de cada temática (Tabla 1). La temática predominante fue la simetría de una kata y fue definida como el porcentaje de técnicas repetidas con ambos lados del cuerpo. Una kata fue considerada simétrica si tenía más del 20\% de repetición de técnicas con ambos lados del cuerpo.

Sólo se utilizaron las katas clásicas, ya que éstas son las formas que se han enseñado para ser transmitidas de Higaonna Kanryo a Miyagi Chojun. Por lo tanto, las katas Sanchin, Tensho, y Gekisai no fueron incluidas en este análisis. Tensho y Gekisai fueron excluidas puesto que son ejercicios modernos, mientras que Sanchin tiene prácticamente una simetría completa.

\section{TABLA I: Existencia de patrones en las katas clásicas Goju-ryu}

NOTA: Sanchin = patrón Sanchin; Seisan = patrón Seisan; Tres repeticiones $=$ comienzo de la kata con tres repeticiones; Patrón de cruz = patrón en cuatro direcciones; Maegeri = patada frontal; Kansetsugeri $=$ patada lateral baja; Nidangeri $=$ patada doble; Otras patadas $=$ otras patadas; Patadas asimétricas $=$ patadas realizadas sólo con la pierna izquierda; Simetría $=>20 \%$ de repetición por ambos lados.

\begin{tabular}{|c|c|c|c|c|c|c|c|c|}
\hline $\begin{array}{c}\text { Temáticas I } \\
\text { Kata }\end{array}$ & Sanseru & Seisan & Suparimpei & Saifa & Seiunchin & Shisochin & Seipai & Kururunfa \\
\hline I. Sanchin & 1 & 1 & I & 0 & 0 & 1 & 0 & 0 \\
\hline 2. Seisan & 0 & 1 & 1 & 0 & 0 & 0 & 0 & 1 \\
\hline $\begin{array}{l}\text { 3. Tres } \\
\text { repeticiones }\end{array}$ & 1 & 1 & I & I & I & I & 0 & 0 \\
\hline $\begin{array}{l}\text { 4. Patrón de } \\
\text { Cruz }\end{array}$ & 1 & 1 & I & 0 & 0 & 1 & 0 & 0 \\
\hline 5. Maegeri & 1 & 1 & 1 & 1 & 0 & 1 & I & 1 \\
\hline 6. Kansetsugeri & 1 & 1 & 0 & 0 & 0 & 0 & 0 & 0 \\
\hline 7. Nidangeri & I & 0 & I & 0 & 0 & 0 & 0 & 0 \\
\hline $\begin{array}{l}\text { 8. Otras } \\
\text { patadas }\end{array}$ & 1 & 1 & I & 0 & 0 & 0 & 0 & 0 \\
\hline $\begin{array}{l}\text { 9. Patadas } \\
\text { asimétricas }\end{array}$ & 1 & 1 & 1 & 0 & 0 & 0 & 0 & 0 \\
\hline 10. Simetría & 0 & 0 & 0 & I & I & I & I & I \\
\hline
\end{tabular}

Posteriormente se realizó un análisis de conglomerados. El análisis de conglomerados permite la clasificación de diferentes cosas u objetos en grupos similares (conglomerados). El análisis separa cada kata en un grupo diferente de modo que una kata es más parecida a otras pertenecientes a su grupo en comparación con otras katas fuera de su grupo. Esto permite la separación de la información en conjuntos significativos que comparten características comunes. El análisis de conglomerados forma una estructura en forma de árbol conocida como un dendograma para mostrar visualmente las agrupaciones de diferentes cosas, en este caso katas. El análisis de conglomerados se realizó utilizando el programa estadístico SPSS (V14.0)3. Estas técnicas permitieron la clasificación de las katas Goju-ryu para comprender mejor su estructura. 


\section{Resultados y discusión}

El análisis de conglomerados basado en la categorización de temáticas en la Tabla 1 reveló que las katas clásicas formaban dos conglomerados (Figura 1). El primer conglomerado incluiría Sesan, Suparimpei/Pechurin, y Sanseru. El segundo conglomerado incluiría Saifa, Shisochin, Seipai, Kururunfa, y Seiunchin. El primer conglomerado fue etiquetado como el Conglomerado Higaonna (Conglomerado H). El segundo conglomerado fue etiquetado como el Conglomerado Miyagi (Conglomerado M). Esto hizo surgir algunas preguntas importantes en relación a la evolución del Goju-ryu moderno tal y como fue formulado por Miyagi Chojun.

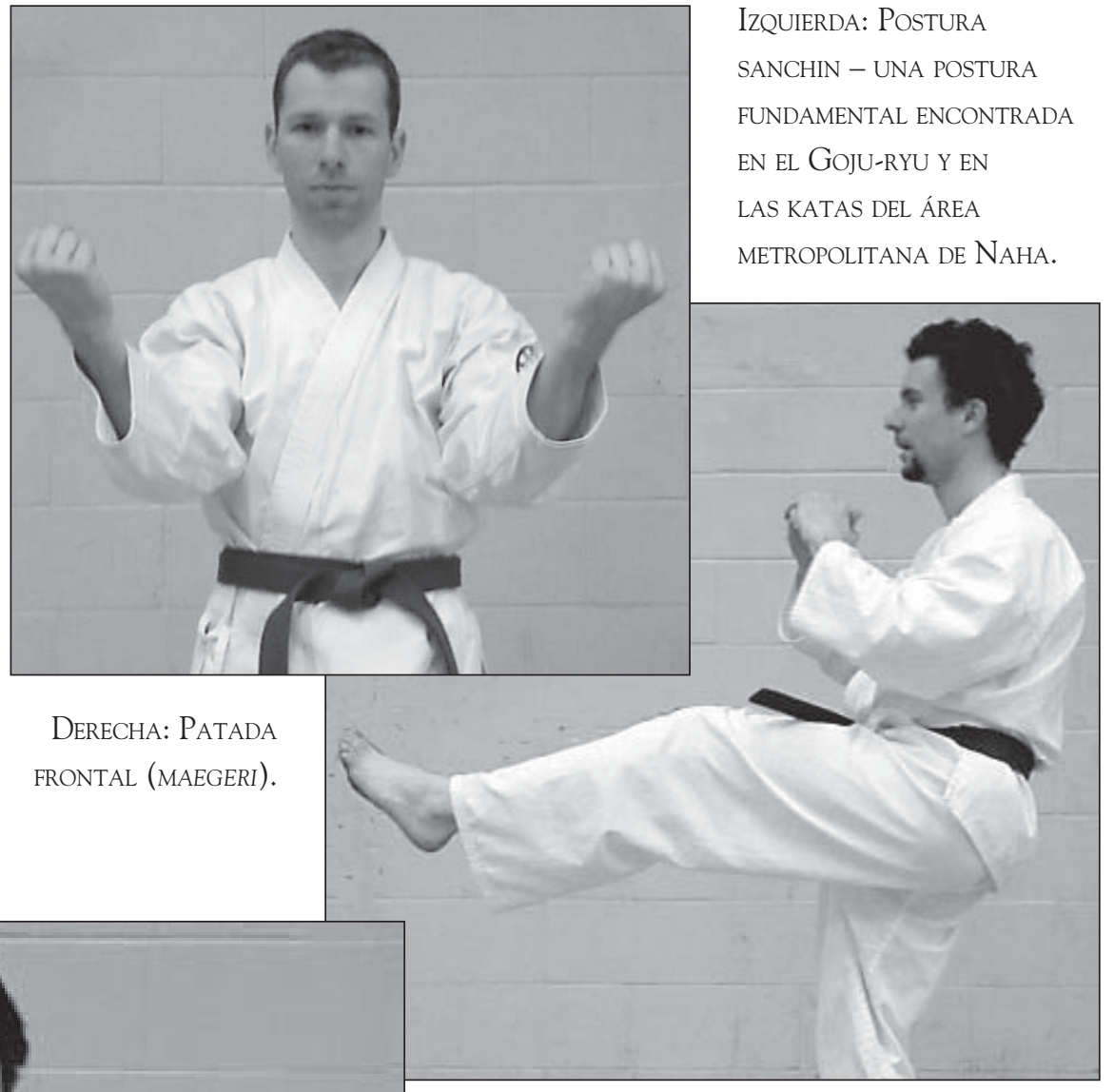

Debajo: Patada a la Rodilla (Kansetsugeri).

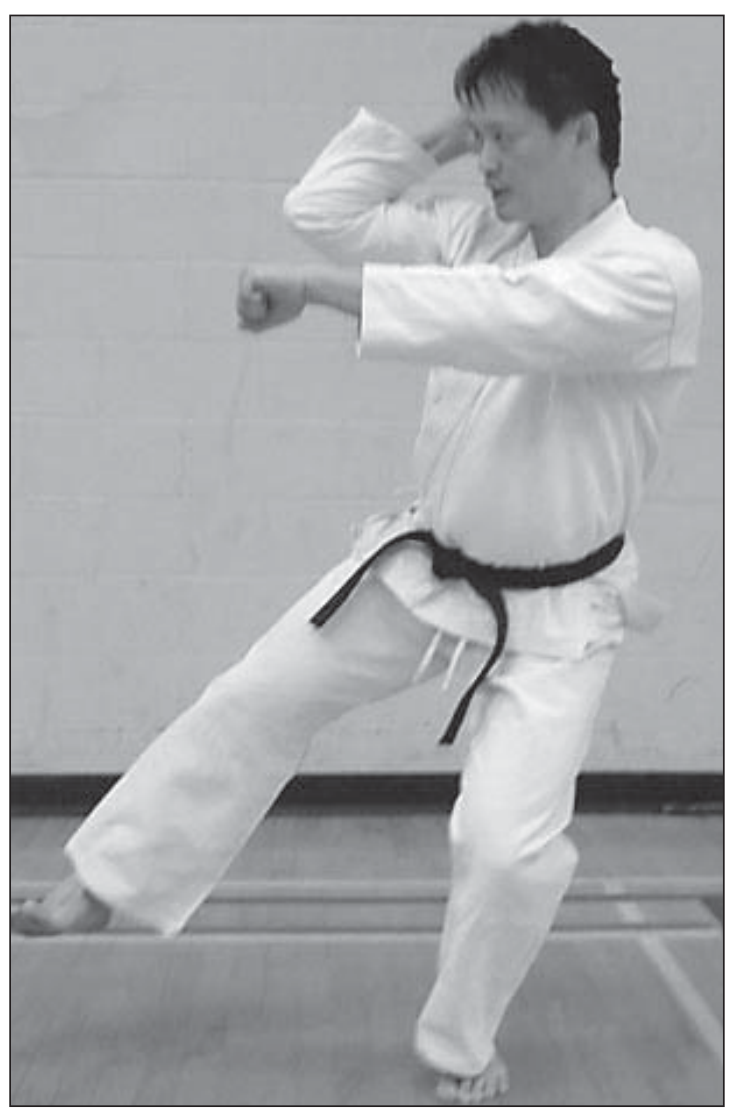

EnCima: Movimiento de APERTURA DE LA KATA SEIUNChin. 
FIGURA I

Diagrama de árbol para las ocho katas

clásicas de Goju-ryu

Enlace completo, distancias euclídeas cuadradas

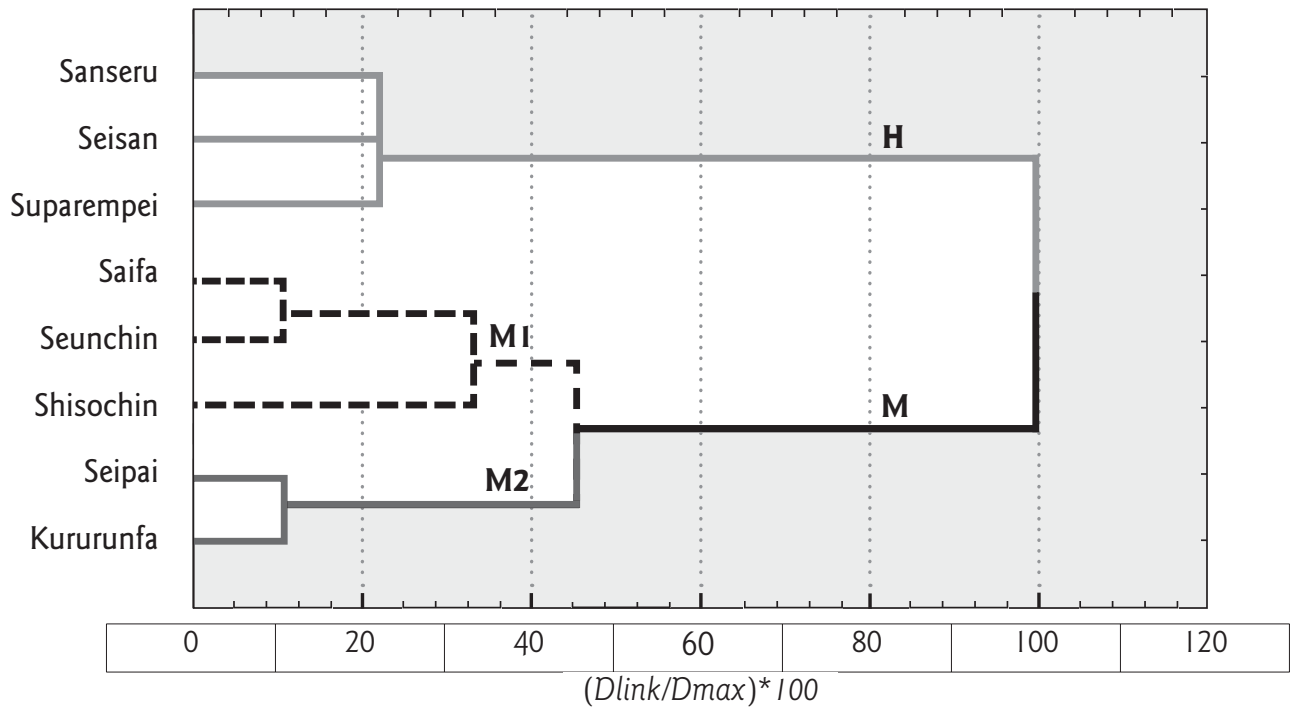

La tabla superior representa visualmente los resultados del análisis de conglomerados. Se trata de un dendograma o un diagrama en forma de árbol. Esto muestra la relación entre las diferentes katas y los grupos que forman. Podemos ver que el Higaonna o Conglomerado H (línea gris) consta de Sanseru, Seisan y Suparimpei/Pechurin. En contraste podemos ver que el Miyagi o Conglomerado M (línea negra) consta de Saifa, Seiunchin, Shisochin, Seipai y Kururunfa. Observando más de cerca podemos ver que el análisis descompone el Conglomerado M en dos grupos más pequeños: Conglomerado MI (línea rayada) el cual contiene Saifa, Seiunchin y Shisochin, y el Conglomerado M2 (línea negra 70\%), que contiene Seipai y Kururunfa.

Primero, ipodría considerarse el Conglomerado $\mathrm{H}$ un método de boxeo indígena mientras que el Conglomerado M podría considerarse boxeo chino? El 19 de marzo de 1866, el último grupo de mensajeros chinos visitaron Okinawa. Documentado con el programa de esta última visita, hubo una demostración de boxeo y armamento por Aragaki Seisho $^{4}$ y otros en el Ochayagoten ${ }^{5}$, en el castillo de Shuri. Se enumeraban diversos nombres de katas incluyendo Seisan y Suparimpei/Pechurin (McKenna, 2001). Ambas katas son parte del Conglomerado H. Por lo tanto, estas dos katas fueron practicadas en Okinawa antes de que supuestamente Higaonna Kanryo partiese a China, y así podrían considerarse indígenas. Podemos suponer que debido al agrupamiento de las katas en el Conglomerado H, que Sanchin y Sanseru también podrían haber sido practicadas durante esta época, sugiriendo que el Conglomerado $\mathrm{H}$ representa un método de boxeo de Okinawa. Se puede ver una evidencia que lo corrobora más aún si examinamos el currículo del To'on-ryu de Kyoda Juhatsu, el cual contiene sólo cuatro katas: Sanchin, Seisan, Sanseru y Pechurin. Así, podemos especular que el Conglomerado H podría representar las katas originales de Nahate (Sanchin, Seisan, Sanseru, y Suparimpei/Pechurin) como defendían Kanzaki y Murakami.

Segundo, estos resultados sugieren que Higaonna Kanryo fue el responsable de la introducción del Conglomerado $\mathrm{H}$, pero no existe una prueba clara de que fuera el responsable de la introducción del Conglomerado M. La otra kata encontrada en el Conglomerado M parece ser más una adicción a aquel currículo que una parte integral del mismo. También podemos decir que el Conglomerado $\mathrm{M}$ aparece por primera vez como parte de las enseñanzas de Miyagi Chojun.

Podría discutirse que las cuatro katas originales del Conglomerado $\mathrm{H}$ fueron formas para principiantes y sólo Miyagi Chojun aprendió las otras formas más avanzadas que se encuentran en el Conglomerado M. Sin embargo, como señala Hirakami (2001), observando el contenido técnico de las cuatro katas encontradas en el Conglomerado $\mathrm{H}$, es difícil mantener esta teoría. Por lo tanto, es interesante teorizar sobre que el 
Conglomerado M representa katas chinas más nuevas introducidas por Miyagi Chojun después de la muerte de Higaonna en 1915.

Por último, si Higaonna Kanryo enseñó las katas que forman el Conglomerado M sólo a Miyagi Chojun, entonces Higaonna transmitió dos o más sistemas diferentes. Esto es, Higaonna transmitió una versión modificada de lo que constituyen las artes de combate originales de la zona metropolitana de Naha como representan las katas Sanchin, Sesan, Sanseru, y Suparimpei/Pechurin. Esto entonces suscita la pregunta, ¿de dónde vienen estas katas adicionales? ¿Se originaron a partir del maestro de Higaonna en China, Ru Ru Ko, o quizás vienen de otra persona?

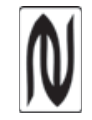

\section{NOTAS}

${ }^{1}$ Los caracteres utilizados por Itosu para referirse al kárate pueden pronunciarse toudi en el dialecto de Okinawa, significando "Mano de Tang/China" mostrando un origen o influencia china.

2 Aunque esto se contradice en el libro de Morio Higaonna, The History of Karate: Okinawan Goju-ryu [La historia del kárate: el Goju-ryu de Okinawa] (1998).

${ }^{3}$ En el SPSS (V14.0), el análisis de conglomerados utiliza la relación media como un algoritmo para la fusión y las distancias euclídeas al cuadrado.

${ }^{4}$ Aragaki Seisho (1840-1920) está considerado el primer maestro de boxeo de Higaonna Kanryo.

${ }^{5}$ La casa real del té situada en el castillo de Shuri.

\section{BIBLIOGRAFÍA - INGLÉS}

Higaonna, M. (1998). The history of karate: Okinawan Goju-ryu. Thousand Oaks, CA: Dragon Books.

McKenna, M. (2001). Exploring goju ryu's past: Myths \& facts surrounding Higashionna Kanryo, pt. 1-2. Dragon Times, 18-19.

McCarthy, P. (1993). An outline of karatedo. International Ryukyu Karate Research Society.

\section{BIBLIOGRAFÍA - JAPONÉS}

HIRAKAMI, N. (2001, mayo). Secret of Nafadi and Fujian boxing, pt. 1-2. Gekkan Hiden, 110-114.

HOKAMA, T. (1999). Okinawa karate-do kobudo no shinzui. Naha: Naha Shuppansha.

IWAI, T. (1992). The ancient transmission of karate-jutsu. Tokyo: Aiyudo.

KINJO, A. (1999). A true record of the transmission of karate. Okinawa: Tosho Center.

KINJO, H. (2007). Seitokukai Homepage. http://skrt.s43.xrea.com/karatejp/modules/ xoopsfaq/index.php?cat_id=7

MURAKAMI, K. (1991). The heart and technique of karate. Tokyo: Shinjin Butsu Orai Sha Hakko.

OKINAWA PREFECTURAL BOARD OF EDUCATION. (1994). A basic investigative report into karatedo and kobudo. Ginowan: Yojusha.

TOKASHIKI, I. (1991). Gohaku-kai yearbook (Vol. 4). Naha: n.p.

\section{AGRADECIMIENTOS}

Gracias a Fred Lohse III por revisar y editar el manuscrito; y a Justin Chin, Maik Hassle, y Oliver Riche por posar para las fotografías. 